\title{
Estudo da alimentação dos peixes no rio Grande à jusante da usina hidrelétrica de Itutinga, Minas Gerais, Brasil
}

\author{
Cíntia V. Gandini, Igor A. Boratto, Daniela C. Fagundes \& Paulo S. Pompeu
}

Setor de Ecologia, Departamento de Biologia, Universidade Federal de Lavras, Caixa Postal 3037, 37200-000, Lavras, MG, Brasil. (cgandini@yahoo.com.br)

\begin{abstract}
Study of fish diet at Grande river downstream from Itutinga hydroelectric power plant, Minas Gerais, Brazil. The aquatic biota of the Paraná river basin has been impacted by several dams. This study aimed characterize and compare seasonally the fish feeding of Grande river fish fauna, downstream from Itutinga Power Plant. In this river stretch it is expected the reduction of seasonal effects due to flow regulation. Data collection was conducted in January and July 2010 with gill nets and trawls. The fishes were fixed in formalin and preserved in alcohol for stomach contents analysis. The diet was characterized by Feeding Index (IA) and the guilds each species belongs was determined by the $\chi^{2}$ test. The feeding of each species and the occurrence of food items were compared in periods of drought and rain using nMDS and SIMPER analysis. We analyzed 809 stomachs from 32 fish species categorized in piscivorous, herbivorous, ilyophagous/detritivorous, ilyophagous, algivorous, frugivorous, invertivorous and generalists guilds; the generalists had the highest species richness. The nMDS indicated lower diet diversification in July, when it was observed an increase of plant, invertebrate and sediment occurrences. However, differences in diet were lower than expected. This study expanded knowledge about fish feeding habits and local dam effects on species diet as a result of flow regulation.
\end{abstract}

KEYWORDS. Reservoir, feeding habit, tropical fishes, diversity.

RESUMO. A biota aquática da bacia do rio Paraná tem sido impactada por várias barragens. Este estudo teve como objetivo caracterizar e comparar sazonalmente a alimentação dos peixes do rio Grande, à jusante da usina de Itutinga. Neste trecho de rio é esperada a redução do efeito sazonal devido à regulação do fluxo. A coleta foi realizada em janeiro e julho de 2010, com redes de emalhar e de arrasto. Os peixes foram fixados em formol e conservados em álcool para a análise dos conteúdos estomacais. A dieta foi caracterizada pelo índice alimentar (IA) e a guilda a que cada espécie pertence foi determinado pelo teste de $\chi^{2}$. A alimentação de cada espécie e a ocorrência dos itens alimentares foram comparadas nos períodos de seca e chuva utilizando-se as análises de nMDS e SIMPER. Foram analisados 809 estômagos de 32 espécies de peixes categorizadas nas guildas piscívora, herbívora, iliófaga/detritívora, algívora, frugívora, invertívora e generalista, sendo esta a guilda de maior riqueza de espécies. O nMDS indicou menor diversificação na dieta em julho, quando foi observado um aumento na ocorrência de vegetal, invertebrados e sedimento. Este estudo ampliou o conhecimento dos hábitos alimentares dos peixes e o efeito do barramento local sobre a dieta das espécies em virtude da regularização do fluxo.

PALAVRAS-CHAVE. Reservatório, hábito alimentar, peixes tropicais, diversidade.

A construção de hidrelétricas, por meio da regulação do fluxo de água no rio e da retenção de nutrientes no reservatório, modifica a entrada da matéria orgânica à jusante deste sistema (Poff et al., 1997; Agostinho et al., 2009). O barramento pode, por exemplo, reduzir ou impedir a comunicação do rio com as várzeas, restringindo a variabilidade e abundância de recursos alimentares presentes no ambiente (HAHN \& Fugi, 2007; Agostinho et al., 2008).

A construção de barragens na bacia do rio Paraná tem alterado o fluxo natural dos rios e, consequentemente, as funções ecológicas dos ecossistemas aquáticos (Agostinho et al., 2008). O rio Grande, por exemplo, apresenta-se atualmente bastante impactado pela construção de numerosas usinas hidrelétricas ao longo do seu curso. Até o momento, há poucos estudos nessa bacia relacionados aos impactos das usinas sobre a ictiofauna da região (CASTRO et al., 2004), em especial sobre aqueles relacionados à alimentação de peixes.

$\mathrm{O}$ estudo da alimentação dos peixes é uma importante ferramenta na determinação da estrutura trófica da comunidade e da disponibilidade dos recursos alimentares em ambientes impactados (AgostinHo et al., 2009; Monteiro et al., 2009), servindo como comparativo para outros estudos. Esse embasamento também é fundamental no fornecimento de estratégias usadas na conservação dos ambientes aquáticos e, consequentemente, na melhoria das condições oferecidas às espécies (Pompeu \& GodinHo, 2003).

Este trabalho teve como objetivo caracterizar e comparar sazonalmente a alimentação das espécies de peixes encontradas no alto rio Grande, à jusante da usina hidrelétrica Itutinga, região sul de Minas Gerais. Considerando que o trecho amostrado está compreendido entre duas barragens próximas, é esperado que o fluxo e a disponibilidade sazonal de recursos alimentares sejam alterados, levando a modificações na dieta das espécies.

\section{MATERIAL E MÉTODOS}

O rio Grande está localizado na região sul do estado de Minas Gerais e, junto ao Paranaíba, é um dos principais formadores do rio Paraná (CEMIG, 2010). Na bacia do rio Grande já foram registradas aproximadamente 105 espécies de peixes, muitas delas são consideradas raras ou endêmicas (PomPeu et al., 2009). Na região do alto rio Grande, existem alguns barramentos como as usinas hidrelétricas de Funil e Itutinga que estão separadas por um trecho remanescente lótico de aproximadamente 20 $\mathrm{km}$ de extensão. A área de estudo está localizada $3 \mathrm{~km}$ à jusante da barragem da Usina Hidrelétrica de Itutinga (44' $\left.39^{\prime} \mathrm{W}, 21^{\circ} 16^{\prime} \mathrm{S} ; 850 \mathrm{~m}\right)$, município de Itutinga, MG (Fig. 1).

Os peixes foram capturados em redes de emalhar 


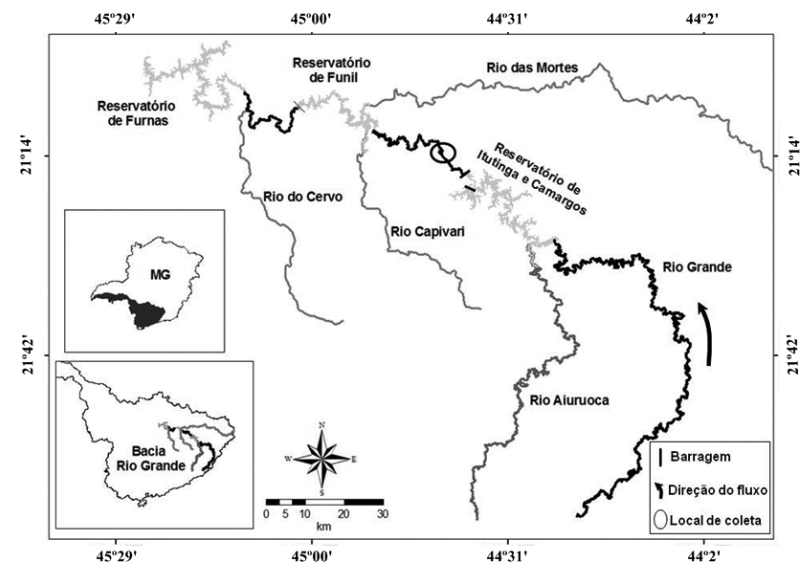

Fig. 1. Mapa da bacia do rio Grande com os principais barramentos e o trecho do rio Grande (círculo preto), à jusante da Usina Hidrelétrica Itutinga, estado de Minas Gerais, onde foram realizadas as amostragens.

(malhas de 2,4 $\mathrm{cm}$ a $16 \mathrm{~cm}$ entre nós) e de arrasto (malha de $4 \mathrm{~mm}$ ) durante seis dias consecutivos de amostragem, nos meses de janeiro e julho de 2010. As redes de espera foram vistoriadas todos os dias a cada doze horas; às seis e 18 horas, enquanto o arrasto foi utilizado todos os dias às seis, 14 e às 22 horas. Os peixes coletados foram fixados em formol $10 \%$ e conservados em álcool $70 \%$. Todos os peixes capturados nas redes de espera tiveram seu conteúdo estomacal analisado. Dos peixes amostrados pela rede de arrasto, a cada dia de coleta, cinco indivíduos de cada espécie e horário foram selecionados para análise do conteúdo estomacal. Estes exemplares foram dissecados para retirada dos estômagos e os itens alimentares foram identificados em estereomicroscópio e pesados individualmente, sendo considerado o peso mínimo de 0,01g. Exemplares de todas as espécies foram depositados na Coleção de Ictiologia da Universidade Federal de Lavras (CI-UFLA 0261 a 0293).

Para caracterização da dieta das espécies foi utilizado o Índice Alimentar (IA) proposto por KAWAKAMI \& VAzzoler (1980), que combina a frequência de ocorrência $(\mathrm{Fi}=$ número de vezes em que o item i ocorreu, dividido pelo número total de estômagos com alimento $)$ e o peso relativo $(\mathrm{Pi}=$ somatório do peso do item i dividido pelo somatório do peso de todos os itens) de cada item, sendo $\mathrm{IA}_{\mathrm{i}}=$ índice alimentar do item $\mathrm{i} ; \mathrm{F}_{\mathrm{i}}=$ frequência de ocorrência do item i e $\mathrm{P}_{\mathrm{i}}=$ peso do item $\mathrm{i}$, na fórmula: $\mathrm{IAi}=\left(\mathrm{F}_{\mathrm{i}} \mathrm{P}_{\mathrm{i}}\right) \sum_{i=1}^{\mathrm{n}} \mathrm{F}_{\mathrm{i}} \mathrm{P}_{\mathrm{i}}$

As espécies com mais de cinco indivíduos analisados foram separadas em grupos tróficos, segundo suas preferências alimentares. Para isso, foi verificado se a proporção do item mais consumido por cada espécie diferia significativamente das demais por meio do teste de qui-quadrado $\left(\chi^{2}\right)$. Quando observadas diferenças significativas, o item mais consumido foi considerado como a dieta preferencial da espécie, permitindo incluíla em uma determinada guilda trófica. $\mathrm{Na}$ ausência de diferenças significativas as espécies foram consideradas generalistas. Confrontando a similaridade entre a frequência de ocorrência dos itens alimentares de cada espécie, nos meses de seca e chuva, comparamos diferenças sazonais na dieta através de nMDS (NonMetric Multidimensional Scaling). A mesma ordena as espécies num espaço multidimensional, utilizando o índice de Bray-Curtis, de acordo com a semelhança de padrões observados para a alimentação. A distância entre as espécies na matriz indica quão similares são suas dietas. A contribuição de cada item alimentar para as diferenças observadas entre estações foi avaliada através da análise de SIMPER.

\section{RESULTADOS}

Foram analisados 809 estômagos de 32 espécies de peixes, pertencentes às ordens Characiformes, Gymnotiformes, Perciformes e Siluriformes, sendo Knodus moenkhausii (Eigenmann \& Kennedy, 1903), Piabina argentea Reinhardt, 1867 e Bryconamericus stramineus Eigenmann, 1908 as mais abundantes (Tab. I).

Os valores significativos para o teste $\chi^{2}$ indicaram a existência de sete guildas tróficas para a comunidade analisada: herbívora, piscívora, iliófaga, iliófaga/ detritívora, algívora, frugívora e invertívora. Seis espécies consumiram itens variados em proporções semelhantes sendo categorizadas como generalistas (Tab. II), guilda com o maior número de representantes (Fig. 2). Para $28 \%$ das espécies, a quantidade insuficiente de estômagos não permitiu a determinação da respectiva guilda trófica.

A análise de nMDS indicou que houve grande sobreposição das dietas nos dois períodos de amostragem, mas no mês de janeiro existiu uma maior variedade de itens componentes da dieta dos peixes, evidenciada pela maior dispersão dos dados (Fig. 3). Muitas espécies apresentaram mudanças na proporção de itens ingeridos entre períodos de coleta, sendo estas mais pronunciadas para Hypostomus margaritifer (Regan, 1908), Astyanax altiparanae Garutti \& Britski, 2000 e Odontostilbe sp. (Fig. 3).

A análise de Simper mostrou que os itens mais consumidos pelos peixes, que foram sedimento, vegetal e invertebrados, tiveram maior frequência média no mês de julho, em comparação com janeiro (Tab. III).

\section{DISCUSSÃO}

A análise dos conteúdos estomacais permitiu observar padrões de dieta e variações no espectro alimentar proporcionadas pelas condições do ambiente no qual os peixes estão inseridos (FUGI et al., 2001; MonTEIRo et al., 2009). Apesar da interferência exercida pelas barragens sobre o fluxo de energia (Agostinho et al., 1992) e da limitação espacial, o presente estudo mostrou que existe grande variedade de guildas tróficas 
Tab. I. Número de estômagos analisados por espécie de peixes coletados em janeiro e julho de 2010, à jusante da usina hidrelétrica Itutinga, no rio Grande, estado de Minas Gerais, Brasil.

\begin{tabular}{|c|c|c|c|}
\hline Táxon & $\begin{array}{c}\text { Estômagos } \\
\text { analisados }\end{array}$ & Táxon & $\begin{array}{l}\text { Estômagos } \\
\text { analisados }\end{array}$ \\
\hline Ordem Characiformes & & Ordem Characiformes & \\
\hline Família Anostomidae & & Família Erythrinidae & \\
\hline Leporellus vittatus (Valenciennes, 1850) & 9 & Hoplias lacerdae Miranda Ribeiro, 1908 & 1 \\
\hline Leporinus amblyrhynchus Garavello \& Britski, 1987 & 14 & Família Parodontidae & \\
\hline Leporinus elongatus Valenciennes, 1850 & 5 & Apareiodon affinis (Steindachner, 1879) & 51 \\
\hline Leporinus friderici (Bloch, 1794) & 10 & Parodon nasus Kner, 1859 & 1 \\
\hline Leporinus octofasciatus Steindachner, 1915 & 20 & Família Prochilodontidae & \\
\hline Leporinus striatus Kner, 1858 & 1 & Prochilodus lineatus (Valenciennes 1836) & 3 \\
\hline Schizodon nasutus Kner, 1858 & 27 & Ordem Gymnotiformes & \\
\hline Família Characidae & & Família Sternopygidea & \\
\hline Astyanax altiparanae Garutti \& Britski, 2000 & 15 & Eigenmannia virescens (Valenciennes, 1836) & 8 \\
\hline Astyanax fasciatus (Cuvier, 1819) & 38 & Ordem Perciformes & \\
\hline Astyanax scabripinnis (Jenyns, 1842) & 1 & Família Cichlidae & \\
\hline Astyanax aff. paranae Eigenmannn, 1914 & 1 & Cichlasoma paranaense Kullander, 1983 & 1 \\
\hline Bryconamericus stramineus Eigenmann, 1908 & 158 & Geophagus brasiliensis (Quoy \& Gaimard, 1824) & 11 \\
\hline Galeocharax knerii (Steindachner, 1879) & 6 & Ordem Siluriformes & \\
\hline Knodus moenkhausii (Eigenmann \& Kennedy, 1903) & 133 & Família Loricarridae & \\
\hline Odontostilbe sp. & 12 & Hypostomus aff. margaritifer (Regan, 1908) & 8 \\
\hline Piabina argentea Reinhardt, 1867 & 171 & Hypostomus $\mathrm{sp} 1$ & 17 \\
\hline Serrapinnus sp. & 1 & Família Pimelodidae & \\
\hline Serrapinnus heterodon (Eigenmann, 1915) & 12 & Iheringichthys labrosus (Lütken, 1874) & 15 \\
\hline Família Crenuchidae & & Pimelodus heraldoi Azpelicueta, 2001 & 1 \\
\hline Characidium aff. zebra Eigenmann, 1909 & 2 & Pimelodus maculatus La Cepède, 1803 & 22 \\
\hline \multicolumn{4}{|l|}{ Família Curimatidae } \\
\hline Cyphocharax nagelii (Steindachner, 1881) & 34 & TOTAL & 809 \\
\hline
\end{tabular}

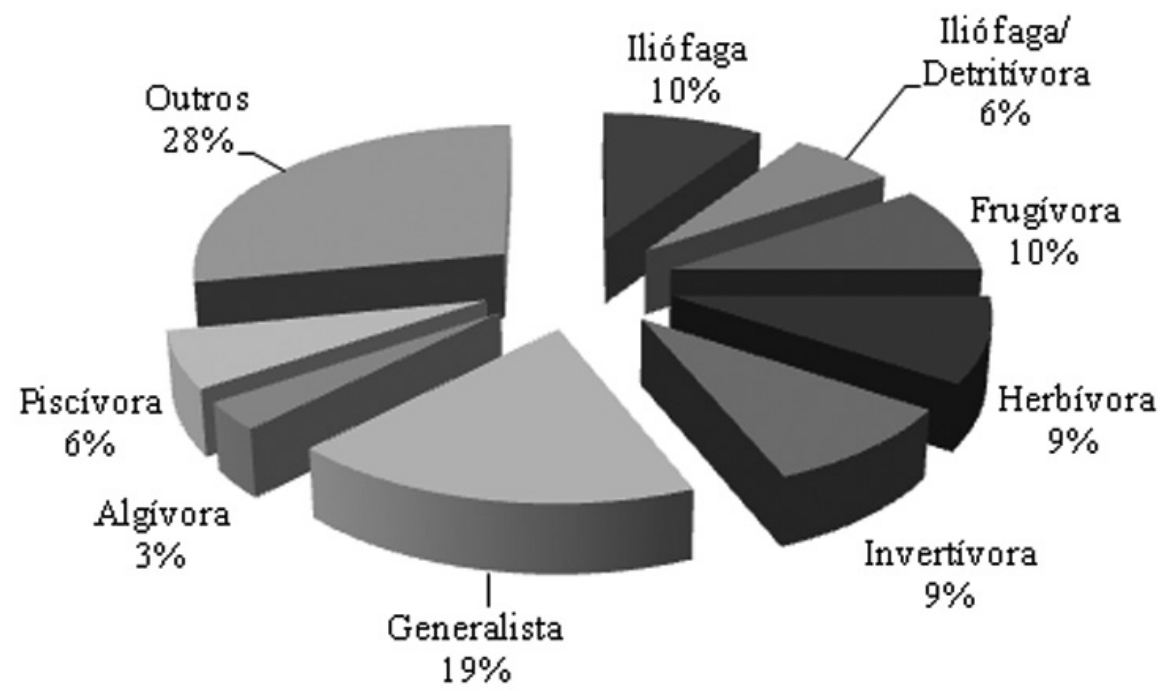

Fig. 2. Riqueza porcentual de espécies representantes das diferentes guildas tróficas (iliófaga, iliófaga/detritívora, frugívora, herbívora, invertívora, generalista, algívora e piscívora) e aquelas que não foram categorizadas em guildas (outros), coletadas em janeiro e julho de 2010 , à jusante da usina hidrelétrica Itutinga, no rio Grande, estado de Minas Gerais, Brasil. 
neste trecho do rio Grande. Mesmo havendo variação sazonal na frequência de ocorrência de determinados itens, as espécies avaliadas praticamente não alteraram a preferência alimentar do período chuvoso para o seco.

Atividades antrópicas nos ambientes naturais, como é caso dos barramentos, podem promover mudanças na estrutura e composição das espécies (Agostinho et al., 2008, 2009). À jusante do reservatório, as principais alterações ocorrem pela regularização do fluxo de água, gerando retenção de nutrientes à montante e perda dos sítios de alimentação, como as planícies de inundação (Craig, 2000; Agostinho et al., 2008). Com a mudança na proporção dos recursos alimentares disponíveis, algumas guildas tróficas podem ser perdidas ou se tornar restritas a poucas espécies (Agostinho et al., 2008). Apesar disso, os resultados aqui obtidos permitem constatar que a maioria dos grupos tróficos representativos para cursos de água doce esteve presente no local estudado, sugerindo que muitas espécies podem manter o seu padrão alimentar diante de alterações ambientais relevantes (AgostinHo et al., 2008).

A morfologia, vazão e atributos físicos e químicos

Tab. II. Valores do Índice Alimentar e teste de qui-quadrado $\left(\chi^{2}\right)$ com determinação de guildas tróficas das espécies coletadas em janeiro e julho de 2010, à jusante da usina hidrelétrica Itutinga, no rio Grande, Minas Gerais, Brasil (1, vegetal; 2, sedimento; 3, fruto; 4, alga; 5, detrito; 6, semente; 7, peixe; 8, invertebrados aquáticos; 9 , invertebrados terrestres; I, iliófaga; F, frugívora; $\mathrm{H}$, herbívora; In, invertívora; $\mathrm{P}$, piscívora; I/D, iliófaga/ detritívora; G, generalista; A, algívora).

\begin{tabular}{|c|c|c|c|c|c|c|c|c|c|c|c|}
\hline \multirow[b]{2}{*}{ Espécie } & \multicolumn{9}{|c|}{ Itens alimentares } & \multirow[b]{2}{*}{ Teste $\chi^{2}$} & \multirow{2}{*}{$\begin{array}{l}\text { Guilda } \\
\text { trófica }\end{array}$} \\
\hline & 1 & 2 & 3 & 4 & 5 & 6 & 7 & 8 & 9 & & \\
\hline Apareiodon affinis & 0,001 & 0,527 & 0,000 & 0,440 & 0,014 & 0,000 & 0,018 & 0,000 & 0,000 & $\mathrm{p}<0,001$ & I \\
\hline Astyanax aff. paranae & 0,000 & 0,333 & 0,000 & 0,333 & 0,000 & 0,000 & 0,000 & 0,333 & 0,000 & & \\
\hline Astyanax altiparane & 0,045 & 0,001 & 0,835 & 0,000 & 0,003 & 0,000 & 0,003 & 0,114 & 0,001 & $\mathrm{p}=0,009$ & $\mathrm{~F}$ \\
\hline Astyanax fasciatus & 0,341 & 0,002 & 0,595 & 0,028 & 0,002 & 0,000 & 0,000 & 0,028 & 0,003 & $\mathrm{p}<0,001$ & $\mathrm{~F}$ \\
\hline Astyanax scabripinnis & 0,333 & 0,333 & 0,000 & 0,000 & 0,333 & 0,000 & 0,000 & 0,000 & 0,000 & & \\
\hline Bryconamericus stramineus & 0,583 & 0,010 & 0,000 & 0,001 & 0,099 & 0,000 & 0,003 & 0,297 & 0,006 & $\mathrm{p}<0,001$ & $\mathrm{H}$ \\
\hline Characidium aff. zebra & 0,000 & 0,125 & 0,000 & 0,000 & 0,125 & 0,000 & 0,000 & 0,750 & 0,000 & & \\
\hline Cichlasoma paranaense & 0,000 & 0,000 & 0,000 & 0,500 & 0,500 & 0,000 & 0,000 & 0,000 & 0,000 & & \\
\hline Cyphocharax nagelii & 0,013 & 0,987 & 0,000 & 0,000 & 0,000 & 0,001 & 0,000 & 0,000 & 0,000 & $\mathrm{p}<0,001$ & I \\
\hline Eigenmmania virescens & 0,015 & 0,110 & 0,000 & 0,007 & 0,007 & 0,000 & 0,000 & 0,860 & 0,002 & $\mathrm{p}<0,001$ & In \\
\hline Galeocharax knerii & 0,000 & 0,000 & 0,000 & 0,000 & 0,000 & 0,000 & 1,000 & 0,000 & 0,000 & $\mathrm{p}<0,001$ & $\mathrm{P}$ \\
\hline Geophagus brasiliensis & 0,063 & 0,448 & 0,000 & 0,007 & 0,343 & 0,000 & 0,000 & 0,140 & 0,000 & $\mathrm{p}=0,255$ & $\mathrm{I} / \mathrm{D}$ \\
\hline Hoplias lacerdae & 0,000 & 0,000 & 0,000 & 0,000 & 0,000 & 0,000 & 1,000 & 0,000 & 0,000 & & $\mathrm{P}$ \\
\hline Hypostomus aff. margaritifer & 0,001 & 0,544 & 0,000 & 0,000 & 0,454 & 0,000 & 0,000 & 0,000 & 0,000 & $\mathrm{p}=0,131$ & $\mathrm{I} / \mathrm{D}$ \\
\hline Hypostomus sp. 1 & 0,000 & 0,967 & 0,000 & 0,000 & 0,032 & 0,000 & 0,000 & 0,000 & 0,000 & $\mathrm{p}<0,001$ & I \\
\hline Iheringichthys labrosus & 0,158 & 0,337 & 0,000 & 0,001 & 0,000 & 0,018 & 0,000 & 0,486 & 0,000 & $\mathrm{p}=0,057$ & G \\
\hline Knodus moenkhausii & 0,164 & 0,210 & 0,001 & 0,254 & 0,152 & 0,000 & 0,000 & 0,219 & 0,001 & $\mathrm{p}>0,050$ & A \\
\hline Leporellus vittatus & 0,215 & 0,109 & 0,000 & 0,000 & 0,001 & 0,000 & 0,000 & 0,710 & 0,001 & $\mathrm{p}=0,014$ & In \\
\hline Leporinus amblyrhynchus & 0,015 & 0,137 & 0,000 & 0,000 & 0,015 & 0,000 & 0,000 & 0,833 & 0,002 & $\mathrm{p}=0,012$ & In \\
\hline Leporinus elongatus & 0,818 & 0,006 & 0,001 & 0,000 & 0,000 & 0,157 & 0,000 & 0,015 & 0,000 & $\mathrm{p}=0,658$ & G \\
\hline Leporinus friderici & 0,637 & 0,017 & 0,298 & 0,000 & 0,031 & 0,000 & 0,018 & 0,037 & 0,000 & $\mathrm{p}=0,111$ & G \\
\hline Leporinus octofasciatus & 0,253 & 0,180 & 0,556 & 0,000 & 0,001 & 0,000 & 0,000 & 0,009 & 0,000 & $\mathrm{p}=0,006$ & $\mathrm{~F}$ \\
\hline Leporinus striatus & 1,000 & 0,000 & 0,000 & 0,000 & 0,000 & 0,000 & 0,000 & 0,000 & 0,000 & & \\
\hline Odontostilbe sp. & 0,061 & 0,435 & 0,000 & 0,435 & 0,007 & 0,000 & 0,000 & 0,061 & 0,000 & $\mathrm{p}=0,326$ & G \\
\hline Parodon nasus & 1,000 & 0,000 & 0,000 & 0,000 & 0,000 & 0,000 & 0,000 & 0,000 & 0,000 & & \\
\hline Piabina argentea & 0,422 & 0,110 & 0,138 & 0,050 & 0,115 & 0,000 & 0,000 & 0,164 & 0,000 & $\mathrm{p}<0,001$ & $\mathrm{H}$ \\
\hline Pimelodus heraldoi & 0,000 & 0,000 & 0,000 & 0,000 & 0,000 & 0,000 & 1,000 & 0,027 & 0,000 & & \\
\hline Pimelodus maculatus & 0,356 & 0,049 & 0,047 & 0,056 & 0,105 & 0,000 & 0,004 & 0,339 & 0,042 & $\mathrm{p}=0,825$ & G \\
\hline Prochilodus lineatus & 0,000 & 1,000 & 0,000 & 0,000 & 0,000 & 0,000 & 0,000 & 0,000 & 0,000 & & \\
\hline Schizodon nasutus & 0,950 & 0,019 & 0,000 & 0,031 & 0,000 & 0,000 & 0,000 & 0,000 & 0,000 & $\mathrm{p}<0,001$ & $\mathrm{H}$ \\
\hline Serrapinus heterodon & 0,133 & 0,408 & 0,000 & 0,208 & 0,133 & 0,000 & 0,000 & 0,115 & 0,000 & $\mathrm{p}=0,467$ & G \\
\hline Serrapinus sp. & 0,000 & 1,000 & 0,000 & 0,000 & 0,000 & 0,000 & 0,000 & 0,000 & 0,000 & & \\
\hline
\end{tabular}


do canal fluvial interferem na variação espacial e temporal do alimento disponível (Esteves \& ARANHA, 1999). É possível que o ambiente restrito e localizado entre as usinas hidrelétricas Itutinga e Funil tenha afetado a disponibilidade dos recursos alimentares ao longo do ano, favorecendo a presença das espécies com pouca ou nenhuma especialização na dieta. Apesar disso, a maior dispersão dos dados na matriz de nMDS para o período chuvoso mostrou que, nesta estação, a variabilidade de itens na dieta dos peixes foi superior comparada à estação seca. As condições de umidade e temperatura na estação chuvosa são mais propícias à formação de frutos e ao processamento da matéria orgânica, aumentando a quantidade de detritos (Dudgeon, 2008; Davies et al., 2008), o que pode ter contribuído para esta elevada amplitude do espectro alimentar.

O efeito sazonal neste estudo não afetou a estrutura trófica da comunidade, mas alterou a frequência de ocorrência de alguns itens, evidenciada através da análise de SIMPER. Mudanças na proporção dos recursos alóctones e autóctones disponíveis e na produtividade primária do sistema são comuns quando comparamos períodos secos e chuvosos (DAvies et al., 2008). A vegetação no entorno do rio Grande é composta em partes por floresta estacional semi-decidual, cujas plantas tendem a perder as folhas na estação seca, aumentando a abundância deste item (SouzA et al., 2003; IBGE, 2004). A baixa turbulência das águas nesta estação também favorece a proliferação de invertebrados (AlLAN \& CASTILLO, 2007; Ceneviva-Bastos \& CAsatti, 2007; Jacobsen et al., 2008) e aumenta a transparência da água, facilitando o uso do substrato por espécies iliófagas (Power, 1983; DuDGEon,
2008). O aumento na disponibilidade destes itens pode ter proporcionado o consumo mais elevado no mês de julho. A tendência de homogeneização no espectro alimentar na seca (observada na figura 2) também é um reflexo disto, pois a maior parte dos indivíduos consumiu os itens mais abundantes em detrimento de outros.

Apesar da influência das usinas hidrelétricas sobre a disponibilidade dos recursos alimentares, diferenças sazonais na dieta foram menores do que as esperadas. Desta forma, as espécies sofreram algumas variações na frequência de consumo dos itens alimentares, sem alterar o padrão alimentar de maneira significativa. Portanto, este estudo contribuiu para o maior conhecimento sobre a preferência alimentar das espécies nas estações seca e chuvosa, diante de alterações antrópicas como a construção de barragens.

Tab. III. Análise de SIMPER complementar ao nMDS (Non-Metric Multidimensional Scaling) mostrando a mudança na contribuição dos itens alimentares para a dissimilaridade entre as dietas nos dois períodos de coleta e a frequência média de ocorrência de cada item nos conteúdos estomacais, para os peixes coletados em janeiro e julho de 2010, à jusante da usina hidrelétrica Itutinga, no rio Grande, estado de Minas Gerais, Brasil.

\begin{tabular}{lccc}
\hline Item & Contribuição & $\begin{array}{c}\text { Frequência } \\
\text { média (JAN) }\end{array}$ & $\begin{array}{c}\text { Frequência } \\
\text { média (JUL) }\end{array}$ \\
\hline Sedimento & 11,260 & 0,502 & 0,591 \\
Invertebrados & 10,180 & 0,386 & 0,463 \\
Vegetal & 9,793 & 0,385 & 0,505 \\
Alga & 7,350 & 0,214 & 0,205 \\
Detrito & 6,304 & 0,250 & 0,139 \\
Fruto & 5,175 & 0,155 & 0,104 \\
Semente & 0,423 & 0,015 & 0,003 \\
Peixe & 0,284 & 0,001 & 0,002 \\
\hline
\end{tabular}

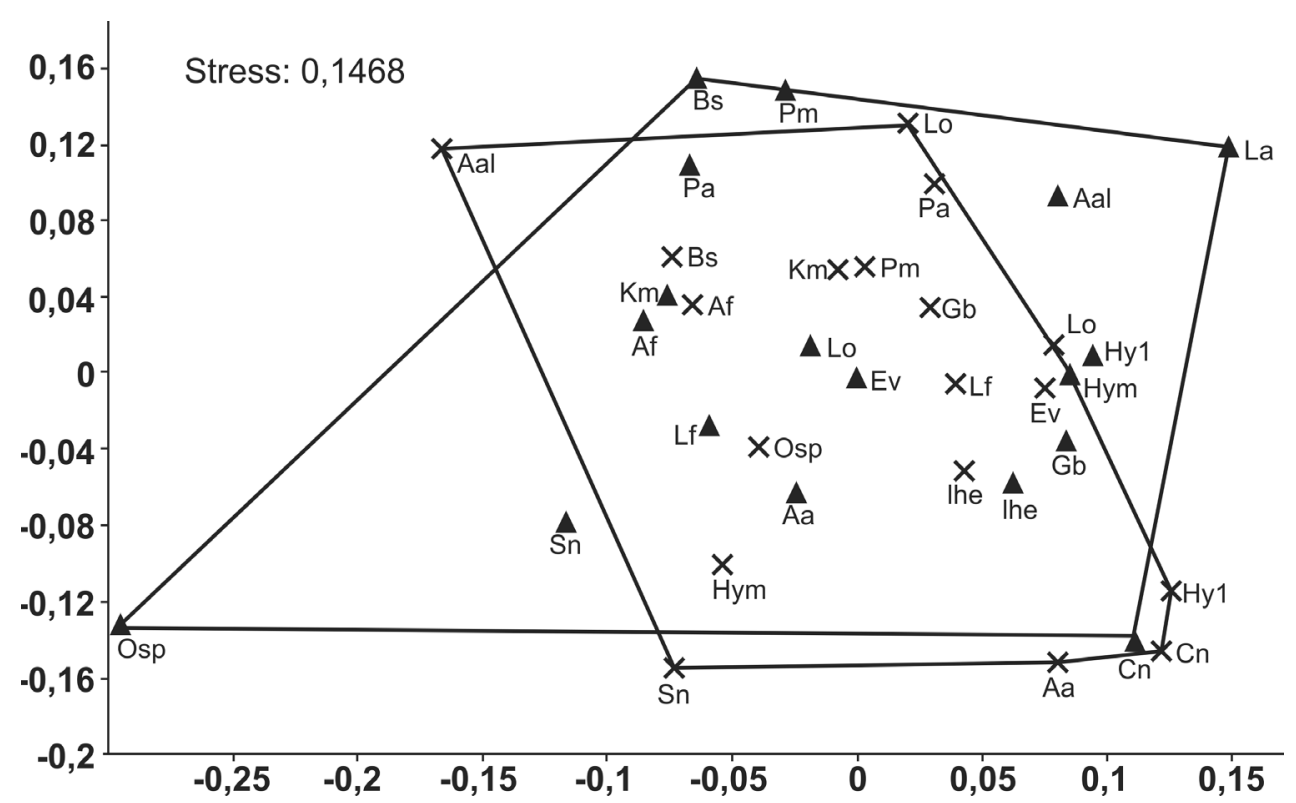

Fig. 3. Análise multivariada nMDS (Non-Metric Multidimensional Scaling) comparando a frequência de ocorrência dos principais itens consumidos pelas espécies de peixes coletadas em janeiro e julho de 2010, à jusante da usina hidrelétrica Itutinga, no rio Grande, estado de Minas Gerais, Brasil ( $\boldsymbol{\Lambda}$, janeiro; X, julho; Aa, Apareiodon affinis; Aal, Astyanax altiparane; Af, Astyanax fasciatus; Bs, Bryconamericus stramineus; Cn, Cyphocharax nagelii; Ev, Eigenmmania virescens; Gb, Geophagus brasiliensis; Hy1, Hypostomus sp. 1; Hym, Hypostomus aff. margaritifer; Ihe, Iheringichthys labrosus; Km, Knodus moenkhausii; La, Leporinus amblyrhynchus; Lf, Leporinus friderici; Lo, Leporinus octofasciatus; Osp, Odontostilbe sp.; Pa, Piabina argentea; Pm, Pimelodus maculatus; Sn, Schizodon nasutus). 
Agradecimentos. Os autores agradecem à CEMIG/ ANEEL pelo financiamento da pesquisa e concessão de bolsas; aos pesquisadores do Laboratório de Macroinvertebrados Bentônicos (UFMG), pelo auxílio na identificação dos conteúdos estomacais; à bióloga Ludmilla Zambaldi (UFLA), pela confecção do mapa e aos companheiros do Laborátório de Ecologia de Peixes (UFLA), pela ajuda nas coletas.

\section{REFERÊNCIAS BIBLIOGRÁFICAS}

Agostinho, A. A.; Júlio-JR., H. F. \& Borghetti, J. R. 1992. Considerações sobre os impactos dos represamentos na ictiofauna e medidas para sua atenuação. Um estudo de caso: reservatório de Itaipu. Revista UNIMAR 14 (supl.):89-107.

Agostinho, A. A.; Pelicice, F. M. \& Gomes, L. C. 2008. Dams and the fish fauna of the Neotropical region: impacts and management related to diversity and fisheries. Brazilian Journal of Biology 68(4, suppl.):1119-1132.

Agostinho, A. A.; Pelicice, F. M. \& Marques, E. E. 2009. Reservatório de peixe angical: bases ecológicas para o manejo da ictiofauna. São Carlos, RiMa. 188p.

Allan, J. D. \& Castillo, M. M. 2007. Stream Ecology. Structure and function of running Waters. Netherlands, Springer. 436p.

Castro, R. M. C.; Casatti, L.; Santos H. F.; Melo, A. L. A.; Martins, L. S. F.; Ferreira, K. M.; Gibran, F. Z.; Benine, R. C.; Carvalho, M.; Ribeiro, A. C.; Abreu, T. X.; Bockmann, F. A.; Pelição, G. Z.; Stopiglia, R. \& Langeani, F. 2004. Estrutura e composição da ictiofauna de riachos da bacia do rio Grande no estado de São Paulo, sudeste do Brasil. Biota Neotropica 4(1):57-95.

CEMIG. 2010. Rios de Minas. Bacia do rio Grande. Disponível em: $<$ www.portalpeixevivo.com.br/rios.asp >. Acesso em: 10.11.2010.

Ceneviva-Bastos, M. \& CAsatti, L. 2007. Oportunismo alimentar de Knodus moenkhausii (Teleostei, Characidae): uma espécie abundante em riachos do noroeste do Estado de São Paulo, Brasil. Iheringia, Série Zoologia, 97(1):7-15.

CRAIG, J. F. 2000. Large dams and freshwater fish biodiversity. In: Berkamp, G.; McCartney, M.; Dugan, P.; McNeely, J. \& ACreman, M. eds. Dams, ecosystem functions and environmental restoration. Disponível em: <http://intranet. iucn.org/webfiles/doc/archive/2001/IUCN913.pdf>. Acesso em: 18.01.2011.

Davies, P. M.; Bunn, S. E. \& Hamilton, S. K. 2008. Primary Production in Tropical Streams and Rivers. In: Dudgeon, D. ed. Tropical Stream Ecology. Oxford, Elsevier. p.23-42.

Dudgeon, D. 2008. Tropical Stream Ecology. Oxford, Elsevier. 370p.
Esteves, K. E. \& Aranha, J. M. R. 1999. Ecologia trófica de peixes de riachos. In: Caramaschi, E. P.; Mazzoni, R. \& Peres-Neto, P. R. eds. Ecologia de peixes de riachos. Série Oecologia Brasiliensis, 6. Rio de Janeiro, PPGE-EFRJ. p.157-182.

Fugi, R.; Agostinho, A. A. \& Hahn, N. S. 2001. Trophic morphology of five benthic-feeding fish species of a tropical floodplain. Revista Brasileira de Biologia 61:27-33.

HAHn, N. S. \& FugI, R. 2007. Alimentação de peixes em reservatórios brasileiros: alterações e consequências nos estágios iniciais do represamento. Oecologia Brasiliensis 11(4):469-480.

IBGE. 2004. Cartas e mapas. Disponível em: <ftp://ftp.ibge.gov.br/ Cartas_e_Mapas/Mapas_Murais/>. Acesso em: 08.09.2010.

Jacobsen, D.; Cressa, C.; Mathooko, J. M. \& Dudgeon, D. 2008. Macroinvertebrates: Composition, Life Histories and Production. In: Dudgeon, D. ed. Tropical Stream Ecology. Oxford, Elsevier. p.65-105.

KawaKami, E. \& Vazzoler, G. 1980. Método gráfico e estimativa de Índice Alimentar aplicado no estudo de alimentação de peixes. Brazilian Journal of Oceanography 29(2):205-207.

Monteiro, A. de S.; Oliveira, A. H. M.; Pelicice, F. M. \& Oliveira, R. J. 2009. Alterações na disponibilidade de recursos alimentares na dieta das principais espécies de peixes. In: Agostinho, C. A.; Pelicice, F. M. \& Marques, E. E. eds. Reservatório de peixe angical: bases ecológicas para o manejo da ictiofauna. São Carlos, RiMa. p.77-86.

Poff, N. L.; Allan, J. D.; Bain, M. B.; Karr, J. R.; Prestegard, K. L.; Richter, B. D.; Sparks, R. E. \& Stromberg, J. C. 1997. The Natural Flow Regime. A paradigm for river conservation and restoration. BioScience 47(11):769-784.

Pompeu, P. S. \& Godinho, H. P. 2003. Dieta e estrutura trófica das comunidades de peixes de três lagoas marginais do médio São Francisco. In: Godinho, H. P. \& Godinho, A. L. orgs. Águas, peixes e pescadores do São Francisco das Minas Gerais. Belo Horizonte, PUC Minas. p.183-194.

Pompeu, P. dos S.; Reis, L. S. dos; Gandini, C. V.; Souza, R. C. R. DE \& FAVERO, J. M. DEL. 2009. The ichthyofauna of upper rio Capivari: defining conservation strategies based on the composition and distribution of fish species. Neotropical Ichthyology 7(4):659-666.

Power, M. E. 1983. Grazing responses of tropical freshwater fishes to different scales of variation in their food. Environmental Biology of Fishes 9(2):103-115.

Souza, J. S.; Santo, F. del Bon; Fontes, M. A. L.; Oliveira Filho, A. T. \& Botezelli, L. 2003. Análise das variações florísticas e estruturais da comunidade arbórea de um fragmento de floresta semidecídua às margens do rio Capivari, Lavras, MG. Revista Árvore 27(2):185-206. 